\title{
Reviewer Acknowledgements
}

Applied Finance and Accounting [AFA] would like to acknowledge the following reviewers for their assistance with peer review of manuscripts for this issue. Many authors, regardless of whether AFA publishes their work, appreciate the helpful feedback provided by the reviewers. Their comments and suggestions were of great help to the authors in improving the quality of their papers. Each of the reviewers listed below returned at least one review for this issue.

\section{Reviewers for Volume 4, Number 2}

Amira Houaneb, University Ibn Khaldoun, Tunisia

Anastasia Kopaneli, University of Patras, Greece

Andrey Kudryavtsev, The Max Stern Yezreel Valley Academic College, Israel

Augustine Akhidime, Benson Idahosa University, Nigeria

Desti Kannaiah, James Cook University, Singapore

Fabio Rizzato, University of Turin, Italy

Gheorghe Morosan, Stefan Cel Mare University Suceava Romania, Romania

Ioan Bogdan Robu, Alexandru Ioan Cuza University of Iasi, Romania

Jayendra S. Gokhale, Embry-Riddle Aeronautical University, USA

Lingesiya Kengatharan, University of Jaffna, Sri Lanka

Marco Muscettola, Independent researcher, Italy

Mohammad Sami Ali Al-Dahrawi, Zarqa University, Jordan

Mojeed Idowu John Odumeso-Jimoh, Noble Integrated Resources \& Management, Nigeria

Nikolay Patonov, European Polytechnical University, Bulgaria

Peibiao Zhao, Nanjing University of Science and Technology, China

Rui Fernandes, Porto Accounting and Business School, Portugal

Sawsan Saadi Halbouni, Canadian University Dubai, UAE

Volodymyr Vysochansky, Uzhhorod National University, Ukraine

\section{Angelia Evelyn}

Editorial Assistant

On behalf of,

The Editorial Board of Applied Finance and Accounting

Redfame Publishing

9450 SW Gemini Dr. \#99416

Beaverton, OR 97008, USA

E-mail: afa@redfame.com

URL: http://afa.redfame.com 